\title{
Self-Correlations of Electroencephalograms
}

\section{Acedo*}

Instituto de Matemática Multidisciplinar

Universitat Politècnica de València, Building $8 G, 2^{\text {nd }}$ Floor

46022, Valencia, Spain

\section{F. Aranda ${ }^{\dagger}$}

Laboratorio de Salud Pública

Secretaria de Salud Pública de Bogotá D.C.

Kra. 32 No 12-81, Building LSP, $3^{\text {rd }}$ Floor, Bogotá, Colombia

*luiacrod@imm.upv.es, †arandalozanodiego@gmail.com

A susceptible-infected-susceptible (SIS) cellular automaton model for collective neural interactions proposed recently is revisited. In this model, neurons are simple network nodes with different states: active or firing, and quiescent. The main thesis of this approach is that the electroencephalogram (EEG) could emerge as the fluctuations in the number of firing neurons. In this framework, EEG is understood as a statistical epiphenomenon. In this paper, the mean number of active sites and the self-correlation function both in the SIS stochastic model and in elementary cellular automata (ECAs) are considered. Damped oscillatory relaxation to the stationary state is found both in the SIS model and in ECA rule 30; periodic oscillations are found for other class 3 and class 4 cellular automata. A statistical analysis of the selfcorrelations in real EEG shows that the damped oscillatory relaxations are found both in delta and alpha waves. The normalized amplitude of these correlations is predicted by cellular automata models. This reinforces the view of the brain as a highly complex cellular automata system.

\section{Introduction}

Hans Berger, a German medical doctor, discovered in 1929 that between electrodes attached to the human scalp, a potential difference in the millivolt range could be detected by means of a precision galvanometer [1]. He observed that a rhythmic pattern with a frequency of 8 to $12 \mathrm{~Hz}$ was recorded from subjects with their eyes closed (known as the alpha rhythm or Berger's wave). After opening their eyes, the frequency increased to 12 to $30 \mathrm{~Hz}$ (beta rhythm).

In his work, Berger was inspired by the findings of the surgeon Richard Caton, who in 1875 measured electrical potentials on the cortex of laboratory animals. The discovery of intracranial measures of electrical activity preceded Berger's epoch-making discovery by half a century. The importance of Berger's method is that, as an 
extracraneal and non-invasive technique, it could develop into a very useful monitoring and diagnostic tool for neurologists and psychiatrists.

Electroencephalogram (EEG) discovery was ignored for almost a decade. Most researchers thought that these small currents were artifacts of the experimental apparatus and the human body. Finally, this pioneering work was publicly recognized in 1937 when Berger was invited to an international psychology conference in Paris. The same year, A. Lee Loomis and his collaborators classified the different stages of sleep in relation with EEG signals [2]. At that time, it was clear that during sleep, the EEG pattern underwent changes that could be recognized by counting the number of zero crossings (as a qualitative measure of frequency) and their amplitude. The transitions as proposed today by the American Academy of Sleep Medicine are as follows:

- Stage 1: As observed by Berger, during awareness the brain produces the alpha wave with a mixture of frequencies in the 8 to $12 \mathrm{~Hz}$ range. This is a fast rhythm with low amplitude. When the individual becomes drowsy, the pattern changes to the theta wave with lower frequencies in the 4 to $7 \mathrm{~Hz}$ range.

- Stage 2: In this stage, the basal EEG is sporadically interrupted by Kcomplexes (high-voltage peaks around $100 \mu \mathrm{V}$ occurring with a periodicity of 1.0 to 1.7 minutes) and the so-called sleep spindles or sigma waves with a frequency of 13 to $17 \mathrm{~Hz}$ appear every 0.5 to 1.5 seconds.

- Stage 3: This is the most deep stage of sleep. It is characterized by delta waves ranging from 0.5 to $2 \mathrm{~Hz}$ and an amplitude from the negative to the positive peak around $75 \mu \mathrm{V}$. The small frequency of these waves has led to the name slow-wave sleep for this stage.

Frequencies of EEG patterns are commonly associated with a subcortical pacemaker located at the thalamus. This has been criticized recently as a fallacy in EEG research [3]. An explanation of EEG must resort to models of brain dynamics. One of the most evident and still unexplained features of EEG during the transition through the stages of sleep is the negative correlation between amplitude and frequency, that is, amplitude increases when the subject becomes drowsy and generates theta waves and, subsequently, the subject falls deeply asleep, resulting in delta waves that are recorded by the EEG. It seems paradoxical that when the individual is in the deep sleep state and the global brain activity is apparently smaller that the amplitude of EEG becomes larger. Explaining this phenomenon should be an objective of any theory of brain dynamics. The susceptible-infected-susceptible (SIS) cellular automaton model provides an explanation in terms of a phase transition as the probability of infection $\alpha$ approaches the threshold $\beta N[4,5]$.

Cellular automata models that relate EEG with statistical fluctuations of the neural network could provide an approach to this unsolved problem. In this paper, we explore the self-correlation behavior of EEG in comparison with the solution of the stochastic cellular 
automaton model of the brain. The structure of the paper is as follows. In Section 2, we describe the SIS cellular automaton model of the brain and an exact solution by means of modal series is found by means of the technique in $[6,7]$. In this section, the self-correlation function is also calculated for the SIS model. A classification of the behavior for elementary cellular automata (ECAs) is also performed. In Section 3, the self-correlations of EEG signals are calculated and discussed in relation to the solution of the SIS model and ECAs. The paper ends with some conclusions in Section 4.

\section{The Brain as a Stochastic Cellular Automaton Model}

In previous works, a cellular automaton model for the evolution of the number of firing neurons in the brain at a given time has been proposed $[4,5]$. This model follows the rules of the SIS model from mathematical epidemiology [8], where the quiescent neurons play the role of susceptible individuals and the firing neurons correspond to the infected sites. In the homogeneous mixing regime, every individual is connected to any other individual in the network (complete graph) and the probability for a given site for changing its state from susceptible to infected under the influence of an infected site in the network is a constant, $\alpha$. We must take into account that susceptible sites have a chance of being infected by any infected site in the network because we assume homogeneous mixing (every node is connected to every other node). Therefore, the probability for a susceptible site not being infected by any infected site in the network is $(1-\alpha)^{I(t)}$. Consequently, the probability for a susceptible individual to become infected at the next time step is given by the complementary as

$$
\mathcal{P}(S \rightarrow I)=1-(1-\alpha)^{I(t)} \approx \alpha I(t),
$$

where the approximation is valid for small values of the infection probability, $\alpha$. However, in order to avoid the overcounting of probabilities (a site being infected two times by another site), it has been shown by M. A. M. de Aguiar et al. [9] that this approximation is indeed the correct answer to the problem of infection by multiple neighboring sites. The recovery from the disease proceeds with a constant probability $\beta$. In this case, the infected sites again become susceptible. Taking these rules into account, we can easily write the following mean-field evolution equation for $I(t)$ :

$$
I(t+1)=I(t)+\alpha I(t)(N-I(t))-\beta I(t) .
$$

Alternatively, we can write this equation in terms of the fraction of infected sites, $x(t)=I(t) / N$, as follows:

$$
x(t+1)=x(t)+(N \alpha-\beta) x(t)-N \alpha \mathrm{x}^{2}(t) .
$$


The critical points of this Markovian process are $x_{c}=0$ or $x_{c}=(N \alpha-\beta) / N \alpha$. The trivial point $x_{c}=0$ is stable for $N \alpha<\beta$. If $\alpha \geq \alpha_{c}=\beta / N$, the second point is stable and corresponds to a positive fraction of infected sites. An exact solution of the susceptibleinfected-recovered-susceptible (SIRS) epidemic model has recently been found by using a modal series expansion $[6,7]$. We now implement this technique in the discrete domain to solve equation (3). We propose a modal series for $x(t)$ of the form

$$
x(t)=\sum_{k=0}^{\infty} \mathcal{A}_{k} a^{k t} .
$$

By inserting this expression into the evolution equation in equation (3), we find

$$
\begin{aligned}
& \sum_{k=0}^{\infty} \mathcal{A}_{k} a^{k(t+1)}= \\
& \sum_{k=0}^{\infty} \mathcal{A}_{k} a^{k t}(1+N \alpha-\beta)-N \alpha \sum_{j=0}^{\infty} \sum_{l=0}^{\infty} \mathcal{A}_{j} \mathcal{A}_{l} a^{(j+l) t} .
\end{aligned}
$$

We can reorder the double sum as a Cauchy product by introducing the index $k=j+l$, which runs from 0 to $\infty$. So, we have

$$
\sum_{j=0}^{\infty} \sum_{l=0}^{\infty} \mathcal{A}_{j} \mathcal{A}_{l} a^{(j+l) t}=\sum_{k=0}^{\infty}\left(\sum_{j=0}^{k} \mathcal{A}_{j} \mathcal{A}_{k-j}\right) a^{k t} .
$$

This equation must be satisfied for every $k$, so we obtain a set of infinite equations for the coefficients:

$$
\mathcal{A}_{k} a^{k}=\mathcal{A}_{k}(1+N \alpha-\beta)-N \alpha \sum_{j=0}^{k} \mathcal{A}_{j} \mathcal{A}_{k-j} .
$$

In particular, for $k=0$ we obtain the equation with a solution that is the fixed point of the SIS model:

$$
\mathcal{A}_{0}=\mathcal{A}_{0}(1+N \alpha-\beta)-\mathcal{A}_{0}^{2} N \alpha,
$$

whose solutions are

$$
\mathcal{A}_{0}=0 \text { or } \mathcal{A}_{0}=\frac{N \alpha-\beta}{N \alpha} .
$$

The first solution corresponds to the extinction of the epidemic, which happens for $\alpha<\alpha_{c}=\beta / N$. In this case, the trivial solution is a stable state. For $\alpha>\alpha_{c}$, the stable state is given by the second solu- 
tion, that is, $\mathcal{A}_{0}=\left(\alpha-\alpha_{c}\right) / \alpha$. Notice that, as is obvious, $\mathcal{A}_{0}>0$ for $\alpha>\alpha_{c}$ corresponding to a positive number of active sites.

In the following, we assume that $\alpha>\alpha_{c}$ and $\mathcal{A}_{0}$ is given by the nonzero value. The deduction is very similar for $\mathcal{A}_{0}=0$. For $k=1$, we obtain the following from equation (5):

$$
\mathcal{A}_{1} a=\mathcal{A}_{1}(1+N \alpha-\beta)-2 \mathcal{A}_{0} \mathcal{A}_{1} N \alpha .
$$

We now take into account that $\mathcal{A}_{1} \neq 0$ because this value should correspond to the initial condition of our problem, as shown later. Consequently, equation (10) yields an expression for the basic mode $a$ :

$$
\begin{aligned}
a & =1+N \alpha-\beta-2 \mathcal{A}_{0} \\
N \alpha & =1-(N \alpha-\beta), \quad N \alpha>\beta,
\end{aligned}
$$

where we have taken into account that $\mathcal{A}_{0}$ is the nonzero solution in equation (9). From equation (7), we now know that for any $k \geq 2$,

$$
\begin{aligned}
\mathcal{A}_{k} a^{k} & =\mathcal{A}_{k}(1+N \alpha-\beta)-N \alpha\left(2 \mathcal{A}_{0} \mathcal{A}_{k}+\sum_{j=1}^{k-1} \mathcal{A}_{j} \mathcal{A}_{k-j}\right) \\
& =\mathcal{A}_{k} a-N \alpha \sum_{j=1}^{k-1} \mathcal{A}_{j} \mathcal{A}_{k-j},
\end{aligned}
$$

where we have used equation (11) in the last step. From equation (12), we obtain the recurrence relation as follows:

$$
\mathcal{A}_{k}=\frac{N \alpha}{a-a^{k}} \sum_{j=1}^{k-1} \mathcal{A}_{j} \mathcal{A}_{k-j}, \quad k=2,3, \ldots
$$

It can be easily shown that equation (14) also holds for $\mathcal{A}_{0}=0$, $\mathcal{A}_{1} \neq 0$, but in this case the fundamental mode is given by

$$
a=1+N \alpha-\beta, \quad N \alpha<\beta .
$$

Notice that in equations (11) and (15), we have $a<1$. This is compatible with the convergence of the modal series in equation (4). Moreover, for $a<0$, the series becomes oscillatory. We have already shown that the best fitting of the EEG model for EEG seems to be just above the critical threshold value $\alpha_{c}=\beta / N$ [5] and this implies $a<0$. So, oscillatory damping in the mean activity of the brain must also be found. In Figures 1 and 2, we plot the exact solution obtained from equations (5) and (14) for some typical values of the parameters. For $N=10000$ and $\beta=0.03$, we obtain an exponential decay behavior for $\alpha=2 \times 10^{-5}$ (with a fundamental mode $a=0.83$ ) and oscillatory damping for $\alpha=2 \times 10^{-4}$. In the latter case, the fundamental mode is negative, $a=-0.97$, and this gives rise to the oscillations. 


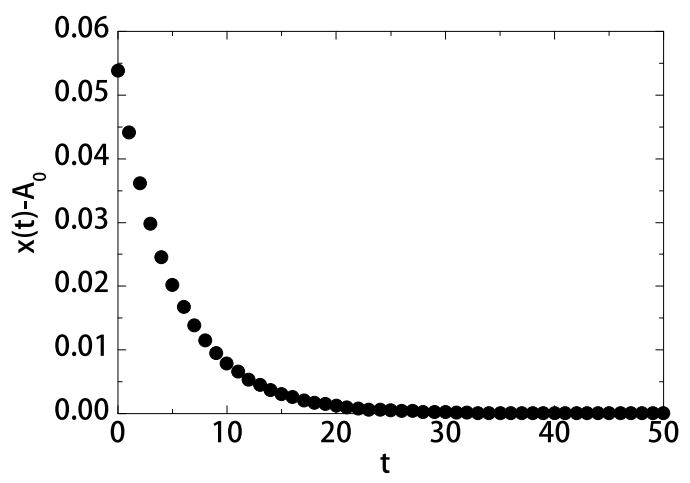

Figure 1. Exact solution of the discrete SIS model for $N=10000$, $\alpha=2 \times 10^{-5}$, and $\beta=0.03$. The stationary state is $\mathcal{A}_{0}=0.85$. The initial condition is $x(t=0)=\mathcal{A}_{0}+0.0538427$ corresponding to $\mathcal{A}_{1}=0.05$. The difference $x(t)-\mathcal{A}_{0}$ is plotted versus time.

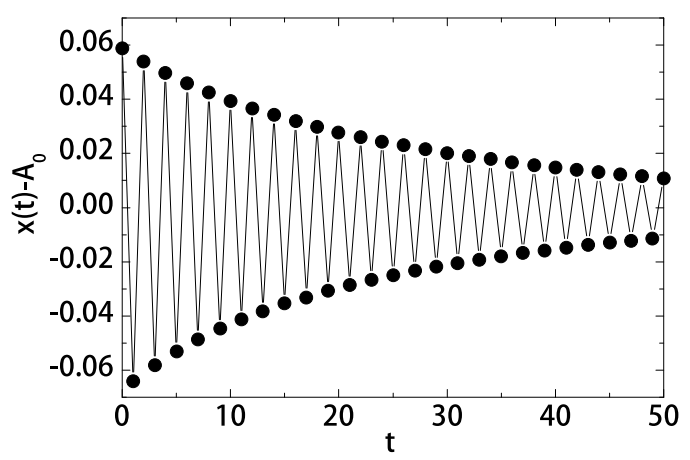

Figure 2. The same as Figure 1 but for $N=10000, \alpha=2 \times 10^{-4}$, and $\beta=0.03$. The stationary state is $\mathcal{A}_{0}=0.985$. The initial condition is $x(t=0)=\mathcal{A}_{0}+0.0588801$ corresponding to $\mathcal{A}_{1}=0.05$.

However, it is not possible to obtain any information about the average number of firing neurons in the brain from EEG because of two reasons: (i) EEG is the potential difference between two electrodes and measures the difference of activity between two brain areas or a brain area and a reference electrode, and (ii) there is no way to perform the average implicit in $x(t)$ from EEG because we do not know if two different points in the record correspond to the same deviation from the stationary asymptotic activity.

So, we will consider the self-correlation function of the signal defined as follows:

$$
\phi(t)=\left\langle x\left(t \mid x_{0}\right) x_{0}\right\rangle-\langle x(t)\rangle^{2},
$$


where $x_{0}$ is the initial fraction of active sites and $x\left(t \mid x_{0}\right)$ is the activity at time $t$ starting from an initial state with a fraction $x_{0}$ of active sites. The average is performed over all possible initial states. The average activity at any instant is given by the asymptotic value $\mathcal{A}_{0}$.

It has been shown that EEG has a Gaussian histogram and this is predicted by the SIS model:

$$
\mathcal{P}(x)=\frac{1}{\sqrt{2 \pi \sigma^{2}}} e^{-\left(x-\mathcal{A}_{0}\right)^{2} /\left(2 \sigma^{2}\right)},
$$

where $\sigma^{2} \simeq 1 / N$ for $\alpha \gtrsim \alpha_{c}$. The average in equation (16) can be calculated approximately:

$$
\begin{gathered}
\left\langle x\left(t \mid x_{0}\right) x_{0}\right\rangle=\int_{0}^{1} x\left(t \mid x_{0}\right) x_{0} \mathcal{P}\left(x_{0}\right) d x_{0} \simeq \\
\int_{-\chi}^{\chi} x\left(t \mid x_{0}\right)\left(\mathcal{A}_{0}+\epsilon\right) \mathcal{P}\left(\mathcal{A}_{0}+\epsilon\right) d \epsilon,
\end{gathered}
$$

where we have used the approximation $x_{0}=\mathcal{A}_{0}+\epsilon$, which is valid for small values of $\mathcal{A}_{1}=\epsilon$ as deduced from equations (4) and (14). The integral can also be truncated to a small interval $\left(\mathcal{A}_{0}-\chi, \mathcal{A}_{0}+\chi\right)$. For example, we have chosen the values $\alpha=2 \times 10^{-4}, \beta=0.35$, and $N=10000$, which correspond to the mode $a=-0.65$. The integral in equation (18) can now be truncated with $\chi=0.05$ with an error of $10^{-6}$ in the normalization of the Gaussian. Using this data, we have found the results in Figure 3. Damped oscillatory behavior is also found.

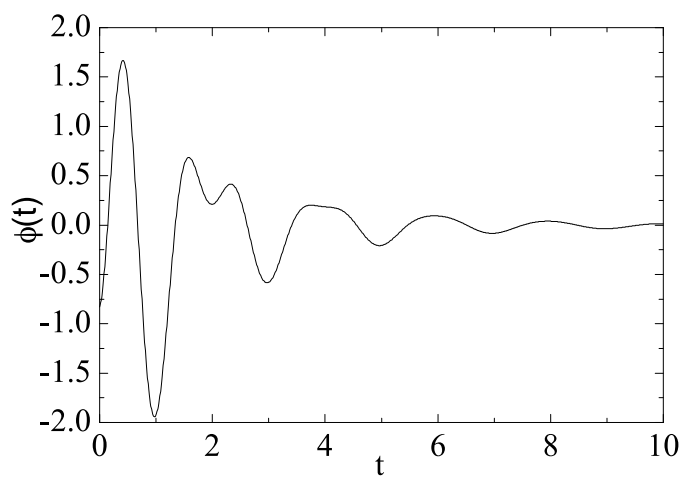

Figure 3. Normalized self-correlation function, $\phi(t) / \mathcal{A}_{0}^{2}$, for the SIS model with $N=10000, \alpha=2 \times 10^{-4}$, and $\beta=0.35$. The solid line interpolation was obtained by taking the real part of equation (18) for non-integer values of time $t$. Values in the vertical axis have been multiplied by a factor $10^{4}$. 
It is useful to investigate how general these behaviors are within the general context of cellular automata models. In particular, we will consider ECA rules [10]. Starting from a random configuration with a certain fraction of active sites (Boolean state 1), we perform simulations for an interval of time and calculate the average number of active sites for every time step. This average is obtained over many evolutions, all starting with the same fraction of active sites but with a distribution of the 1 and 0 states randomly chosen.

Following this method, we observe periodic oscillations in the case of ECA rules 26, 28, 38, 50, and 52. Damped oscillations are found for ECA rule 30 as shown in Figure 4.

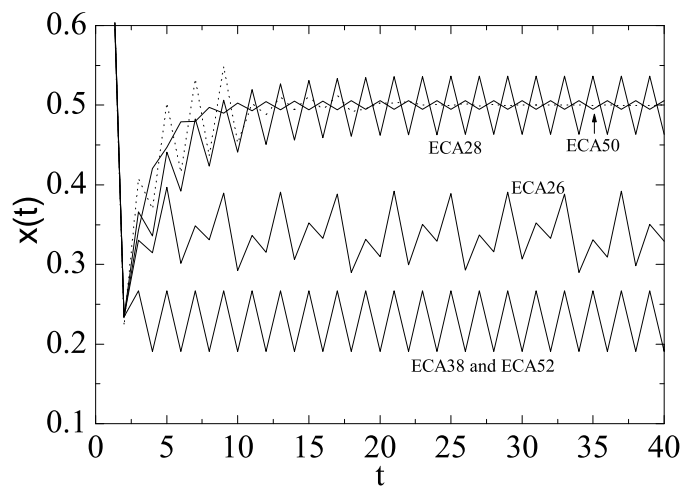

Figure 4. Average number of active sites for ECAs. Solid lines correspond to ECA rules 26, 28, 38, 50, and 52. Dotted line corresponds to ECA rule 30. An average over 5000 simulations of a cellular automaton with 1000 sites was performed.

The self-correlation function for ECA rule 30 can also be calculated if we assume a distribution of initial fractions of active sites. For example, by choosing

$$
\mathcal{P}\left(x_{0}\right)= \begin{cases}0.25 & \text { for } x_{0}=2 / 3 \\ 0.5 & \text { for } x_{0}=1 / 2 \\ 0.25 & \text { for } x_{0}=1 / 3\end{cases}
$$

we obtain the results in Figure 5.

We must also notice that some deterministic versions of the SIS cellular automaton model discussed in this paper have been recently proposed. In particular, Brian's Brain is a deterministic two-dimensional cellular automaton whose rule is as follows: off cells turn on if exactly 2 of their 8 neighbors are on, on cells go into recovery mode, and recovered cells become off [11]. This model displays a sufficient level of complexity that, according to Wolfram's Principle of Computational Equivalence [10], it would be computationally universal. However, 
universality has not been definitely proved. On the other hand, it is obvious that, if the brain is described by a cellular automaton, it should be a computationally universal one because the brain is the paradigm of a general-purpose computational device. In Figure 6, we have plotted the evolution of the SIS rule for a system with 50 nodes.

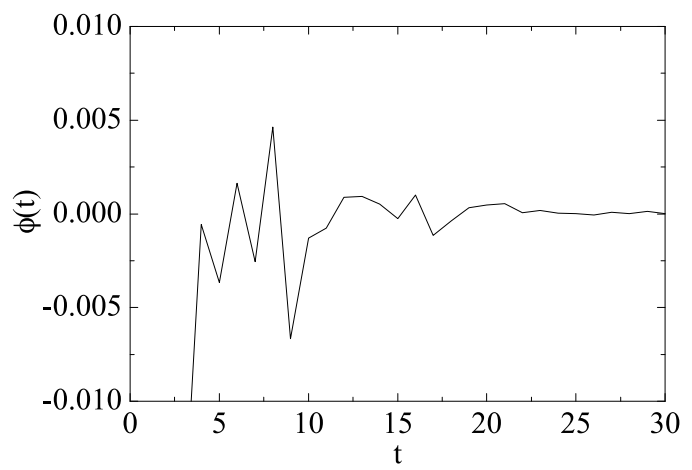

Figure 5. Self-correlations for ECA rule 30. An average over 5000 random initial conditions according to the distribution in equation (19) was performed.

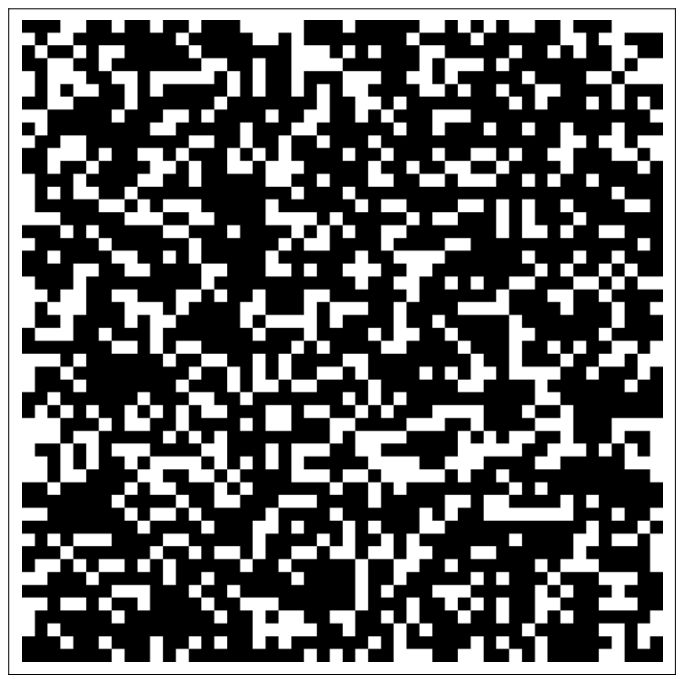

Figure 6. Array plot for the evolution of the SIS rule starting with an initial condition corresponding to $50 \%$ of active sites. Time steps run from top to bottom since $t$ ranges from 0 to 50 . 
The evolution pattern is sufficiently complex to suggest computational universality for the SIS model.

In Section 3, we discuss the relevance of these statistical behaviors for EEG.

\section{Correlation Behavior of Electroencephalograms}

Quantitative EEG analysis is a broad field with many clinical applications [12]. In a pioneering study, N. Wiener proposed that EEG could be understood in terms of a set of nonlinear coupled oscillators [13]. The correlation dimension developed as a tool in the context of the theory of chaotic attractors by Grassberger and Procaccia [14] has been recently used as a way to characterize EEG in different sleep stages [15]. However, the more traditional spectral decay has been found to be more robust [16].

Explaining brain dynamics in terms of chaotic attractors in a deterministic chaotic system is currently losing supporters. The hypothesis of understanding EEG as a fractal structure is more promising. In this context, the widely extended term of "brain waves" to describe the different EEG states has been contributing to the confusion. The epidemic model discussed in this paper provides a scenario in which the erratic activation of nodes in a complete network could mimic the random oscillations of EEG. In every state (awake subject, initiation of sleep, deep sleep, etc.) the brain achieves a stationary state but, as it occurs in the dynamical equilibrium of systems composed by many particles, fluctuations around the mean are observed. In this model, EEG should be a measure of these fluctuations obtained as the difference in the electric potentials of two electrodes attached to the scalp. These electrodes perform an average over many neurons located under the scalp and, consequently, the potential $V(t)$ oscillates randomly depending on the number of active neurons in these areas.

In order to analyze the relaxation of correlations in EEG, we have divided EEG records corresponding to the same state and with a total length that varies from 30 minutes to 1 hour in portions of $10 \mathrm{sec}-$ onds. These portions are then considered as independent records because autocorrelations are supposed to vanish after a few seconds. This is confirmed in the subsequent analysis. The self-correlation function $\phi(t)$ is calculated as follows:

$$
\phi(t)=\langle V(t) V(t=0)\rangle-\langle V(t)\rangle^{2},
$$

where the average is performed over the different portions obtained from the full EEG records and $\langle V(t)\rangle$ denotes the average potential in the full record. Results for slow-wave sleep (delta rhythm) and the alpha rhythm are plotted in Figures 7 and 8, respectively. In both figures, we see that $\phi(t)$ behaves as a damped oscillation. This behavior is particularly conspicuous in the case of the delta rhythm, where a 
more smooth average is obtained. We also see that EEG autocorrelations decay faster for slow-wave sleep than for awake individuals. This is consistent with a larger coordination of different brain areas during wakefulness.

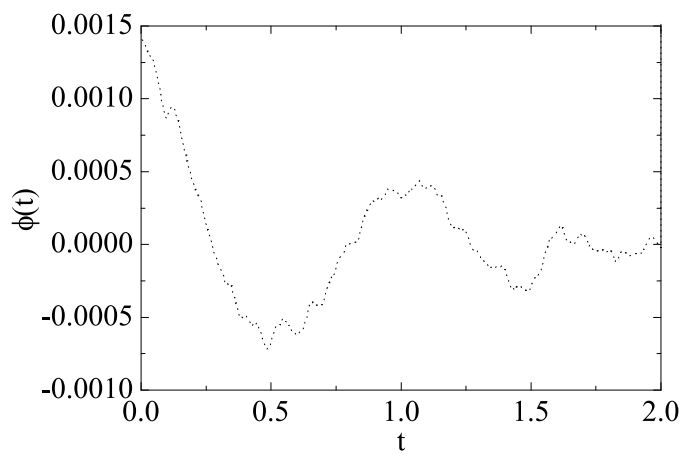

Figure 7. The self-correlation function of the delta rhythm. Time is measured in seconds. $\phi(t)$ is normalized with the average activity $\langle V(t)\rangle^{2}$ in the corresponding data interval.

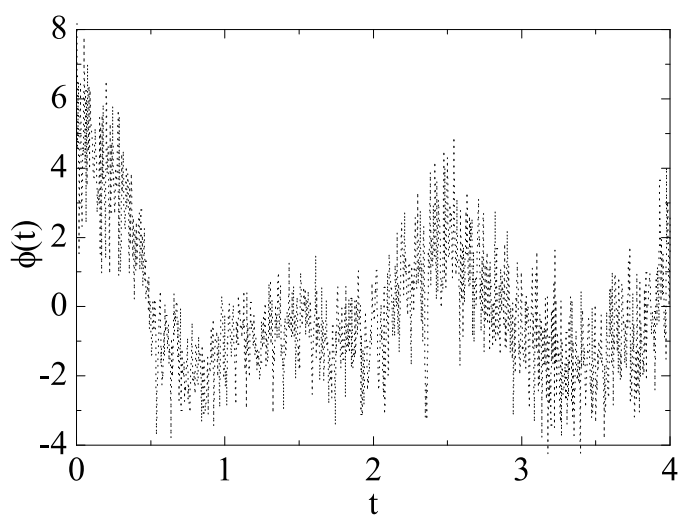

Figure 8. The same as Figure 7 except for the alpha rhythm. The vertical axis represents $\phi(t) /\langle V(t)\rangle^{2}$ in units of $10^{-4}$.

Moreover, damped oscillatory oscillations are common in certain regimes of epidemic models. This is found in the SIS model analyzed in Section 2 and also for ECA rule 30. The order of magnitude of the normalized self-correlations is similar. By comparing Figure 3 and Figure 7 , we can estimate that the underlying brain cellular automaton updates two times per second. A complete oscillation for the cellu- 
lar automaton takes two units of time, which corresponds to one second in the behavior of $\phi(t)$ as shown in Figure 7. This could seem to be a slow update schedule. However, the phi phenomenon (the visual illusion responsible for the merging of the individual images in a film projection into a continuous movie when the brain receives more than 12 images per second) suggests that the visual cortex has a temporal resolution corresponding to the processing of only several images per second [17]. We conclude that the shape of the correlation function of the SIS model is qualitatively similar to that of EEG data from alpha and delta rhythms, and therefore the SIS model warrants further study as a possible mean-field model of neuron dynamics.

\section{Concluding Remarks}

In this paper, we have revisited the susceptible-infected-susceptible (SIS) model for brain dynamics. This model can be understood as stochastic cellular automata with a population of individual automata placed upon the nodes of a complete graph. An interpretation of the SIS model in terms of neural physiology has been proposed: firing neurons correspond with the infected sites, while quiescent neurons are mimicked by susceptible sites. In several previous papers $[4,5]$, this model was investigated in connection with a proposal to understand the electroencephalogram (EEG) as a statistical epiphenomenon, that is, EEG is simulated as the fluctuations in the number of firing (infected) sites in a finite-size complete network evolving according to the SIS model's rules.

In the SIS model of brain activity, firing neurons incite other quiescent neurons in the network to start firing with a probability $\alpha$. The return to the quiescent state for firing neurons is modeled by a Poisson stochastic process with a probability $\beta$ per unit time. The higher amplitude and lower frequencies of the delta wave were found as $\alpha$ decreases because fluctuations become larger in this regime [4]. This could explain the paradox of higher amplitude of EEG signals during deep sleep, where the effectiveness of the propagation of firing activity, $\alpha$, could be lower. This is compatible with the rebound of the levels of glutamic acid, the main excitatory neurotransmitter in the cerebral cortex, observed after sleep deprivation in rats [18-21] or the decline or cerebral glucose utilization during sleep [22].

In this paper, we have shown that the damped oscillatory relaxation behavior that appears in the SIS model and can be fully described analytically is also a pattern found in the EEG signals. This behavior is also found in ECA rule 30.

Cellular automata have already been studied as models of cortical physiology in a rough model by Hofmann [23] and also as a way to disclose spatio-temporal patterns of activity in the hippocampal network [24]. Models of neural populations motivated by the confluence 
of the theory of cellular automata defined upon networks have developed as a field unto itself known as neuropercolation [25].

In our model, we describe the relations between individual automata by means of a complete graph. This is, apparently, a very simple structure for a brain, but a complete set of connections between compartments in the brain has also been proposed as a reasonable model predicting some invariances observed between different species [26].

Nevertheless, the number of synapses that a typical neuron in the human cortex projects toward their neighbors is in the range of 7000 to 12000 [27]. Consequently, a more realistic cellular automaton model of the brain should be defined upon a more sparse network: random network [28] or a Watts-Strogatz network [29]. It would be interesting to analyze the computational capabilities of these cellular automata and their relevance to EEG analysis. Nevertheless, the computational effort to simulate a cellular automaton model in a significantly large system incorporating at least one million nodes is vast and will require the implementation of a distributed computing solution. Work along these lines is in progress.

\section{Acknowledgments}

Financial support from the grant PAID-06-11 Ref: 2087 of the Universidad Politécnica de Valencia is gratefully acknowledged. We also acknowledge the PhysioNet database for providing the EEG records [30].

\section{References}

[1] H. Berger, "Über das Elektroenkephalogramm des Menschen," Archiv für Psychiatrie und Nervenkrankheiten, 87, 1929 pp. 527-570.

[2] A. L. Loomis, E. N. Harvey, and G. A. Hobart, "Cerebral States During Sleep, as Studied by Human Brain Potentials," Journal of Experimental Psychology, 21(2), 1937 pp. 127-144. doi:10.1037/h0057431.

[3] P. L. Nunez and R. Srinivasan, Electric Fields of the Brain-The Neurophysics of EEG, 2nd ed., New York: Oxford University Press, 2006.

[4] L. Acedo, "A Second-Order Phase Transition in the Complete Graph Stochastic Epidemic Model," Physica A: Statistical Mechanics and Its Applications, 370(2), 2006 pp. 613-624. doi:10.1016/j.physa.2006.03.064.

[5] L. Acedo, "A Cellular Automaton Model for Collective Neural Dynamics," Mathematical and Computer Modelling, 50(5-6), 2009 pp. $717-725$. 
[6] L. Acedo, G. González-Parra, and A. J. Arenas, “An Exact Global Solution for the Classical SIRS Epidemic Model," Nonlinear Analysis: Real World Applications, 11(3), 2010 pp. 1819-1825.

doi:10.1016/j.nonrwa.2009.04.007.

[7] L. Acedo, G. González-Parra, and A. J. Arenas, "Modal Series Solution for an Epidemic Model," Physica A: Statistical Mechanics and Its Applications, 389(5), 2010 pp. 1151-1157. doi:10.1016/j.physa.2009.11.003.

[8] N. T. J. Bailey, The Mathematical Theory of Infectious Diseases and Its Applications, 2nd ed., London: Griffin, 1975.

[9] M. A. M. de Aguiar, E. M. Rauch, and Y. Bar-Yam, "Invasion and Extinction in the Mean Field Approximation for a Spatial Host-Pathogen Model,” Journal of Statistical Physics, 114(5-6), 2004 pp. 1417-1451. doi:10.1023/B:JOSS.0000013958.15218.47.

[10] S. Wolfram, A New Kind of Science, Champaign, IL: Wolfram Media, Inc., 2002.

[11] Wikipedia. “Brian’s Brain.” (Jun 11, 2010) http://en.wikipedia.org/wiki/Brian\%27s_Brain

[12] S. Tong and N. V. Thakor, eds., Quantitative EEG Analysis: Methods and Clinical Applications, Boston: Artech House, 2009.

[13] N. Wiener, Nonlinear Problems in Random Theory, New York: John Wiley \& Sons, Inc., 1958.

[14] P. Grassberger and I. Procaccia, "Characterization of Strange Attractors," Physical Review Letters, 50(5), 1983 pp. 346-349. doi:10.1103/PhysRevLett.50.346.

[15] E. Pereda, A. Gamundi, R. Rial, and J. González, "Non-linear Behaviour of Human EEG: Fractal Exponent versus Correlation Dimension in Awake and Sleep Stages," Neuroscience Letters, 250(2), 1998 pp. 91-94. doi:10.1016/S0304-3940(98)00435-2.

[16] A. Krakovska and S. Štolc Jr., "Spectral Decay vs. Correlation Dimension of EEG," Neurocomputing, 71(13-15), 2008 pp. 2978-2985. doi:10.1016/j.neucom.2007.06.007.

[17] M. Wertheimer, "Experimentelle Studien über das Sehen von Bewegung," Zeitschrift für Psychologie und Physiologie der Sinnesorgane, 61, 1912 pp. 161-265.

[18] C. M. McDermott, M. N. Hardy, N. G. Bazan, and J. C. Magee, "Sleep Deprivation-Induced Alterations in Excitatory Synaptic Transmission in the CA1 Region of the Rat Hippocampus," The Journal of Physiology, 570(3), 2006 pp. 553-565. doi:10.1113/jphysiol.2005.093781.

[19] S. Majumdar and B. N. Mallick, "Increased Levels of Tyrosine Hydroxylase and Glutamic Acid Decarboxylase in Locus Coeruleus Neurons after Rapid Eye Movement Sleep Deprivation in Rats," Neuroscience Letters, 338(3), 2003 pp. 193-196.

[20] H. Murck, T. Struttmann, M. Czisch, T. Wetter, A. Steiger, and D. P. Auer, "Increase in Amino Acids in the Pons after Sleep Deprivation: A Pilot Study Using Proton Magnetic Resonance Spectroscopy," Neuropsychobiology, 45(3), 2002 pp. 120-123. doi:10.1159/000054949. 
[21] L. Bettendorff, M. Sallanon-Moulin, M. Touret, P. Wins, I. Margineanu, and E. Schoffeniels, "Paradoxical Sleep Deprivation Increases the Content of Glutamate and Glutamine in Rat Cerebral Cortex," Sleep, 19(1), 1996 pp. 65-71.

[22] P. Maquet, D. Dive, E. Salmon, B. Sadzot, G. Franco, R. Poirrier, R. von Frenckell, and G. Franck, "Cerebral Glucose Utilization During SleepWake Cycle in Man Determined by Positron Emission Tomography and [18F]2-fluoro-2-deoxy-D-glucose Method," Brain Research, 513(1), 1990 pp. 136-143. doi:10.1016/0006-8993(90)91099-3.

[23] M. I. Hofmann, "A Cellular Automaton Model Based on Cortical Physiology,” Complex Systems, 1(1), 1987 pp. 187-202.

[24] M. Tatsuno, Y. Nagai, and Y. Aizawa, "Rule-Dynamical Approach to Hippocampal Network,” Neurocomputing, 38-40, 2001 pp. 965-971. doi:10.1016/S0925-2312(01)00420-9.

[25] R. Kozma, “Neuropercolation,” Scholarpedia, 2(8), 2007 p. 1360. http://www.scholarpedia.org/article/Neuropercolation.

[26] V. Braitenberg, "Brain Size and Number of Neurons: An Exercise in Synthetic Neuroanatomy," Journal of Computational Neuroscience, 10(1), 2001 pp. 71-77. doi:10.1023/A:1008920127052.

[27] M. Abeles, Corticonics: Neural Circuits of the Cerebral Cortex, Cambridge: Cambridge University Press, 1991.

[28] B. Bollobás, Random Graphs, 2nd ed., Cambridge: Cambridge University Press, 2001.

[29] D. J. Watts, Small Worlds: The Dynamics of Networks between Order and Randomness, Princeton, NJ: Princeton University Press, 1999.

[30] A. L. Goldberger, L. A. Amaral, L. Glass, J. M. Hausdorff, P. Ch. Ivanov, R. G. Mark, J. E. Mietus, G. B. Moody, C.-K. Peng, and H. E. Stanley, "PhysioBank, PhysioToolkit, and PhysioNet: Components of a New Research Resource for Complex Physiologic Signals," Circulation, 101(23), 2000 e215-e220.

http://circ.ahajournals.org/cgi/content/full/101/23/e215. 